\title{
Massive pneumonia and pancytopenia leading to the diagnosis of acute lymphoblastic leukemia in child - a case report and a review of the literature
}

\author{
Lorena Elena Melit ${ }^{1,2}$, Cristina Oana Marginean ${ }^{1,2}$, Mihaela Ioana Chincesan ${ }^{1,2}$, \\ Iulia Armean', Vladut Stefanut Sasaran², Maria Oana Marginean ${ }^{1,2}$ \\ ${ }^{1}$ Pediatrics Clinic 1, Emergency County Hospital Targu Mures, Romania \\ 2University of Medicine and Pharmacy Targu Mures, Romania
}

\begin{abstract}
Acute lymphoblastic leukemia (ALL) is one of the most common type of malignancies in children, but at the same time one of the cancers with the best prognostic. Transient pancytopenia has been described to be a very rare entity defined as a preleukemic condition in children and adolescents. We present the case of a 2-year-old male, with a 2-week history of respiratory tract infection, without any improvement after antibiotic therapy, admitted in our clinic with fever, influenced general status, productive cough, intense pallor, palpebral edema and perianal abscess. The laboratory findings revealed severe anemia, mild leukopenia and increased inflammatory biomarkers. The thoracic radiography pointed out a massive right pneumonia. The child was discharge after 3 weeks of wide spectrum antibiotics, presenting a favorable evolution. The follow-up CBC count at approximately 2 weeks from discharge revealed severe leukocytosis, and the immunophenotyping exam of the bone marrow established the diagnosis of pre-B cell type acute lymphoblastic leukemia. All patients treated for pancytopenia must benefit by a proper long-term monitoring in order to rule out the afterwards development of a potential ALL.
\end{abstract}

Keywords: pneumonia, child, acute lymphoblastic leukemia, pancytopenia

\begin{abstract}
Abbreviations
ALL: acute lymphoblastic leukemia AML: acute myeloblastic leukemia BS: body surface

CBC: complete cellular blood count CRP: C-reactive protein

ESR: erythrocyte sedimentation rate H: height
\end{abstract}

\author{
Hb: hemoglobin \\ Htc: hematocrit \\ Leu: leukocytes \\ LRTIs: low respiratory tract infections \\ MCV: medium cellular volume \\ Pre-ALL: preleukemic phase \\ W: weight
}

\section{INTRODUCTION}

Respiratory tract infections which can affect the upper or the lower respiratory tract are probably even the most frequently reported type of infection in human beings (1). Low respiratory tract infections (LRTIs) are a group of disorders that comprise among others pneumonia, and can be one of the most important causes of morbidity and mortality worldwide, especially in pediatric age. Most of the times, LRTIs are mild, transient-lasting and in certain cases self-limiting, and therefore many patients afflicted by these conditions tend to disregard them (2). Thus, under certain conditions, without a proper treatment, they can lead to severe, potentially fatal conditions. LRTIs are very common in 
children, being probably, the first type of infection that occurs after birth, while pneumonia is very often the last condition to develop before death (3). In many cases, the etiological agents of LRTIs cannot be identified and they depend on the geographical area (4). In addition, the etiology, likewise the symptomatology can vary also with other factors, such as: age, gender season, type of population at risk etc. (1).

Leukemia is a relatively rare form of hematological malignancy that occurs when the blood stem cells do not mature adequately leading to their overproduction (4). Although leukemia accounts for approximately only $3 \%$ of adult malignancies, it is one of the most frequently encountered type of cancer in children, representing approximately $15 \%$ of cancers diagnosed under the age of $15(5,6)$. Depending on the type of blood stem cell that is impaired, acute leukemias are divided into two main categories: acute lymphoblastic leukemia (ALL) and acute myeloblastic leukemia (AML). In ALL, the development of lymphoid stem cells is impaired, reaching only the first stages of maturation. Therefore, the lymphoid stem cells can develop into lymphoblasts or at most, into poorly functioning $\mathrm{B}$ or $\mathrm{T}$ lymphocytes that are not able to provide a normal function of the immune system. Furthermore, ALL is divided depending on the type of affected cell lineage into T-cell ALL, if it affect T-lymphocytes (approximately 15\% of the cases), and B-cell type if it impairs B-lymphocytes (the remaining $85 \%$ of the cases) (7). Due to the excessive accumulation of abnormal, leukemic cells in the bone marrow and peripheral blood, the most frequent symptoms encountered in patients with ALL are: frequent infections or other flu-like symptoms, fever, anemia, bleeding, fatigue (7). Nevertheless, certain studies proved that transient pancytopenia can be related to ALL in children and adolescents $(8,9)$. This transient pancytopenic phase is rare and it can occur in approximately $2 \%$ of pediatric ALL cases, being considered a preleukemic condition (pre-ALL)(10). This phase can last from a few weeks to several months and its remission can be spontaneous or induced by corticosteroids, followed by subsequent transformation into common ALL (11). A proper chemotherapy protocol and adequate monitoring can lead to remission in approximately $95 \%$ of the children diagnosed with ALL, and $80 \%$ of those who attaint remission, are expected to be long-term survivors (12). The prognosis of patients diagnosed with ALL is similar after standard chemotherapy independently by the presence or absence of pre-ALL (11).
We report this case of ALL in a small child with the aim of underlining the importance of a proper monitoring after a severe infectious disease, and that the presence of a transient pancytopenic phase can hinder the diagnosis of ALL without an adequate follow-up.

\section{Case report}

\section{Presenting concerns}

We present the case of a 2 year-old male child admitted in our clinic for the following reasons: fever and dry cough for 2 weeks, which became productive for 1 day. The family history did not raise any concerns. We mention also that the patient did not preset any history of toxic exposure (cigarette smoke, radiations, drugs etc.) or other risk factors during the intrauterine life or after birth. The personal history revealed an episode of infectious diarrhea six months ago, an episode of pneumonia (2 months ago) and an episode of pultaceous acute pharyngitis (1 month ago). The onset of the present symptoms was 2 weeks before the admission in our clinic with fever and dry cough initially for which the general practitioner recommended antibiotic and symptomatic treatment, but without any improvements.

\section{Clinical findings}

The clinical exam at the moment of admission revealed the following pathological elements: influenced general status, palpebral edemas, perianal abscess, productive cough, hyperemic pharynx and tonsils, respiratory distress, diminished vesicular murmur on the right side of the thorax, saturation in $\mathrm{O}_{2} 98 \%$, enlarged liver, at $2 \mathrm{~cm}$ under the right costal rib, W: $18.5 \mathrm{~kg}, \mathrm{H}: 95 \mathrm{~cm}, \mathrm{BS}: 0.70 \mathrm{~m}^{2}$.

\section{Diagnostic focus and assessment}

The initial CBC count revealed severe anemia (Hb 5.5 g/dL, Htc 18.5\%, MCV 80.8 fL), mild leukopenia (Leu 3600/ $\mu \mathrm{L}$ ), elevated inflammatory biomarkers (CRP $15.41 \mathrm{mg} / \mathrm{L}$, ESR $105 \mathrm{~mm} / \mathrm{h}$ ). The peripheral blood smear pointed out the predominance of lymphocytes (88\%), hypochromic, microcytic, oval-shaped erythrocytes, and lymphoplasmocytes in ratio of 2/100 leukocytes. The thoracic radiography showed an opacity of subcostal intensity occupying the superior and medium right lobes, establishing the diagnosis of massive right pneumonia (Fig. 1).

We performed also an echocardiography which was normal, and an abdominal ultrasound that pointed out moderate hepatomegaly and abdominal bloating. We solicited a surgical consult for the perianal abscess, who recommended 3-4 times a day washing with antiseptics. The bacteriological exam 


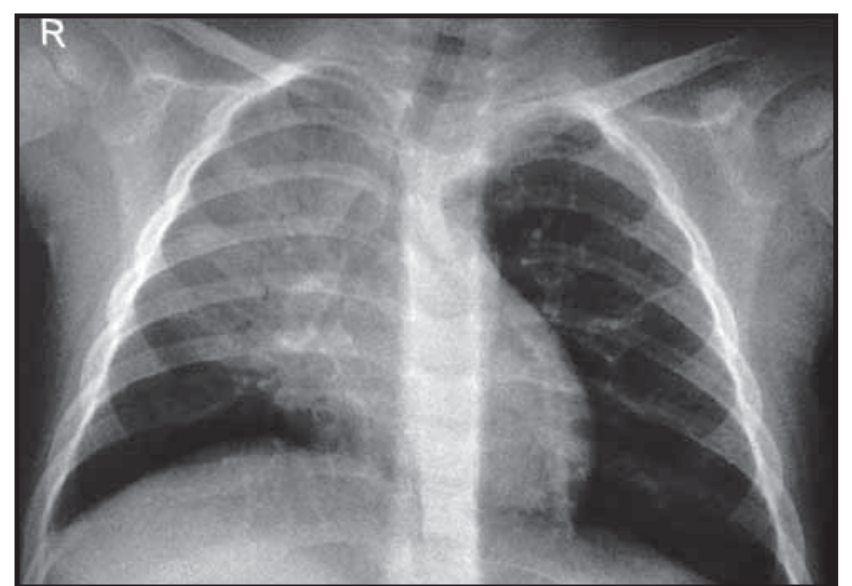

FIGURE 1. Radiological aspect at admission

from the secretion of this abscess revealed Enterococccus faecium. All blood cultures were negative. We ruled out lung tuberculosis based on the negative exam of the gastric aspirate, the presence of post-vaccinal BCG scar and the negative PPD test, establishing the diagnosis of massive right pneumonia and severe anemia.

\section{Therapeutic focus and assessment}

We administered an association of wide spectrum antibiotics (Meronem + Vancomycin), and symptomatic treatment as well, but after 7 days of treatment he developed a tumefaction of the right knee associated with arthralgia and functional impotence. The ultrasound exam showed intraarticular fluid collection suggesting a septic arthritis. The follow-up radiological exam pointed out atelectasis of the medium right lobe (Fig. 2).

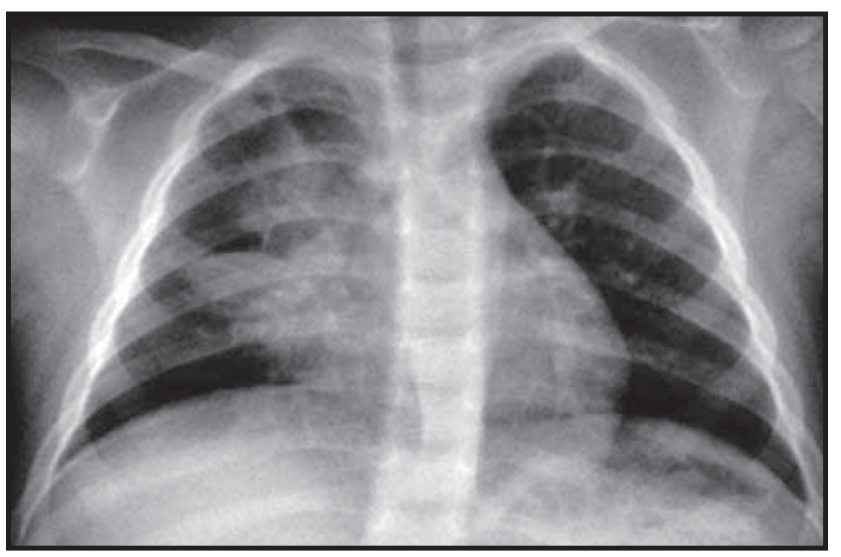

FIGURE 2. Atelectasis of the right medium lobe

Therefore, we performed a bronchoscopy that revealed multiple mucous, viscous secretions the bronchial lumen. We took into account a possible cystic fibrosis, but the sweating test was negative. We continued the antibiotic treatment for another 2 weeks, and we discharged the patient with a mild functional impotence of the right knee and without any pathological laboratory findings.

\section{Follow-up and outcome}

We assessed the patient after 10 days from the moment of discharge and his clinical condition was very good, but the laboratory parameters pointed out an increased number of leukocytes $29,000 / \mu \mathrm{L}$ with lymphocytosis. The peripheral blood smear revealed the following: lymphoblasts $5 \%$, unsegmented cells $2 \%$, segmented cells $25 \%$, monocytes $2 \%$, lymphocytes $66 \%$, a low number of platelets, and an absolute number of blasts 250. Thus, we raised the suspicion of acute lymphoblastic leukemia and we performed a bone marrow exam that showed an increased number of cells in the bone marrow, infiltrated with lymphoblasts (74\%) with a morphologic aspect of $\mathrm{L}_{2}$. We also performed an immunophenotyping exam of the bone marrow that underlined an immunophenotypic aspect of pre-B cell type acute lymphoblastic leukemia. The cytogenetic exam pointed out a normal karyotype, and the molecular biology exam did not reveal any genetic mutations. We also performed the exam of the cerebral spinal fluid which was negative. Based on all these facts, we established the diagnosis of pre-B cell type acute lymphoblastic leukemia with standard risk. We initiated chemotherapy according to the ALL-IC-BFM 2009 protocol, with a favorable evolution, the bone marrow exam from day 15 and 33 indicating the absence of lymphoblasts. Until the present moment, our patient is in complete remission, receiving maintenance cytostatic treatment. The tolerance to chemotherapy was good, without recording major side-effects.

\section{DISCUSSIONS}

Acute lymphoblastic leukemia is one of the most common cancers that occur in children, but despite its increased frequency, more than $90 \%$ of children diagnosed with ALL attaint remission with an encouraging chance of long-term survival (12). Our patient also presented favorable evolution after the administration of standard chemotherapy, reaching complete remission. The type of ALL that originates from B lymphocytes accounts for up to $85 \%$ of the cases, and its subtypes are defined according to the maturity stage of the lymphoblasts, even though the majority comes from precursor B lymphocytes being referred as pre-B cell type ALL (7). Similarly, in our case the immunophenotyping exam revealed an aspect of pre-B cell type ALL. Among others the prognosis of ALL, depends very much on the presence of different chromosomal abnormalities that can be associated either with a good prognosis, either with a worse one. For exam- 
ple, hyperploidy $t(12,21)$ is a chromosomal abnormality associated with a good prognosis, while hyperploidy $t(9,22)$ and mixed-lineage leukemia rearrangements have been documented to have a poor prognosis (13). Fortunately, the genetic exam did not reveal any chromosomal abnormalities in the case we presented above, and therefore we classified the case as presenting standard risk.

The precise trigger of ALL is not established clearly and different hypothesis have been proposed in order to explain the development and onset of the disease. Certain studies underlined the fact that the development of ALL involves genetic disorders, chromosomal abnormalities mostly acquired during fetal hematopoiesis that will result in a subclinical preleukemic clone and/or postnatal secondary genetic changes (14). Nevertheless, the precise event that will determine the preleukemic clones to evolve into an overt ALL remains unidentified (9). Other studies sustain the idea the development of ALL would be attributed to a lack of mobilization of the immune system as a result of insufficient exposure to infectious agents (15). In addition, after the onset ALL will definitely impair the normal functioning of the immune system due to the overproduction of abnormal, immature lymphocytes that cannot fulfill their function. Therefore, in most of the cases, the patients present severe leukocytosis at the onset of the disease suggesting a potential ALL and leading to a biopsy of the bone marrow that will establish the final diagnosis.

Even though it is a well-documented consensus that myelodysplastic syndrome can precede acute non-lymphoblastic leukemia, pre-ALL it is still an unclear, rare entity with a prevalence of $1.3-2.2 \%$ in children diagnosed with ALL $(8,16,17)$. The initial symptom of pre-ALL is fever associated to pancytopenia revealed by the CBC count, and it usually occurs in children under the age of 10 , affecting predominantly girls (18). On one hand, similarly to the previous mentioned data from the literature, the age of our patient was 2 years, but on the other hand he was a boy. Also, Villarreal-Martínez et al. reported two pediatric cases of ALL presenting as aplastic anemia (9). The first one described a 5-year-old male who presented with a perianal abscess whose CBC count revealed a $\mathrm{Hb}$ of $5.0 \mathrm{~g} / \mathrm{dL}$, and also leukopenia and thrombocytopenia. Similarly, the case we described above revealed a boy who presented also a perianal abscess and had a $\mathrm{Hb}$ of $5.5 \mathrm{~g} / \mathrm{dL}$, a mild leukopenia, and a number of platelets within normal limits. The second case reported by the same authors referred to a 2-year-old male, with a 2-week history of upper respiratory tract infection, dark stool, fever, who presented with a $\mathrm{Hb}$ of $1.5 \mathrm{~g} / \mathrm{dL}$, being eventually diagnosed with ALL, as the previous one described by the same authors. Our patient, at the same age as the latter case reported by Villarreal-Martínez et al. presented with a 2-week history of respiratory tract infection. Therefore, it is clear that pre-ALL is a very rare condition that occurs in children and adolescents. Nevertheless, Liang et al. reported a case of 50-year-old female with transient pancytopenia presenting with fever, cough and anemia, who was diagnosed with ALL 3 weeks after the treatment for pancytopenia(18). Similarly, our patient was diagnosed with ALL after approximately 2 weeks from the moment of discharge. Also, in the case described by Liang et al., the laboratory findings revealed severe anemia, neutropenia, and a normal number of platelets, similar to our case. To our best knowledge, this is the $3^{\text {rd }}$ pediatric case reported in the literature that presented with transient pancytopenia as a preleukemic condition, but the first one who associated a massive pneumonia before the onset of ALL.

It is well-documented the fact that ALL can mimic different orthopedic conditions, such as bone, joint or musculoskeletal pain leading to the delay of the proper diagnosis (19). The symptoms of septic arthritis have been frequently encountered in children with ALL, especially those involving the knee or the hip (19). Similarly, our patient presented impairment of the right knee, initially interpreted as septic arthritis, being afterwards defined as a joint involvement in the context of ALL.

Malignant conditions represent a real burden at any age, but especially in pediatrics. Therefore, the early diagnosis and treatment are imperious for increasing the survival rate. Transient pancytopenia - due to its rarity - hinders in most of the cases the diagnosis of acute leukemia leading to a delay in the diagnosis or even misleading the physician into establishing a wrong diagnosis.

\section{CONCLUSIONS}

The clinical and laboratory follow-up of children with severe infections is mandatory in order to rule out different underlying conditions, such as malignancies or immunodeficiency disorders. Therefore, the adequate monitoring of these children can lead to an early diagnosis and treatment of more severe conditions improving their prognosis and their survival rate. Also, transient pancytopenia described as a preleukemic condition, even though rare, must be considered in all cases, and the patient must benefit by a proper long-term monitoring even after its treatment. 


\section{REFERENCES}

1. Khan S., Priti S., Ankit S. Bacteria Etiological Agents Causing Lower Respiratory Tract Infections and Their Resistance Patterns. Iran Biomed J 2015; 19(4):240-6.

2. Ndip R.N., Ntiege E.A., Ndip L.M., Nkwelang G., Akoachere J.F.T.K., Akenji T.N. Antimicrobial resistance of bacterial agents of the upper respiratory tract of school children in Buea, Cameroon. J Health Popul Nutr 2008; 26(4):397-404.

3. Dawadi S., Rao B.S., Khan G.M. Pattern of antimicrobial prescription and its cost analysis in respiratory tract infection. Kathmandu Univ $J$ Sci Eng Technol 2005; I(1):1-9.

4. Anonymous. Leukemia [Internet]. 2015 [cited 2017 Sep 26],. Available from: http://www.llscanada.org/leukemia

5. Canadian-Cancer-Statistics-2014-EN.pdf [Internet]. [cited 2017 Sep 26].. Available from: http://www.cancer.ca/ /media/cancer.ca/CW/ cancer\%20information/cancer $\% 20101 /$ Canadian $\% 20$ cancer $\% 20$ statistics/Canadian-Cancer-Statistics-2014-EN.pdf

6. Howlader N., Noone A.M., Krapcho. SEER cancer statistics review 1975-2010 Bethesda, MD National Cancer Institute Section 28. Bethesda, 2013.

7. Childhood Acute Lymphoblastic Leukemia Treatment [Internet]. National Cancer Institute. [cited 2017 Sep 26],. Available from: https:// www.cancer.gov/types/leukemia/patient/child-all-treatment-pdq

8. Hasle H., Heim S., Schroeder H., Schmiegelow K., Ostergaard E., Kerndrup G. Transient pancytopenia preceding acute lymphoblastic leukemia (pre-ALL). Leukemia 1995; 9(4):605-8.

9. Villarreal-Martínez L., Jaime-Pérez J.C., Rodríguez-Martínez M., González-Llano O., Gómez-Almaguer D. Acute lymphoblastic leukemia of childhood presenting as aplastic anemia: report of two cases. Rev Bras Hematol E Hemoter 2012; 34(2):165-7.

10. Kelly K., Murphy P. Aplastic anaemia preceding acute lymphoblastic leukaemia in an adult with isolated deletion of chromosome 9q. Leuk Res 2008; 32(12):1936-8.

11. Armata J., Grześkowiak-Melanowska J., Balwierz W., NajbarPabian A., Pawlik-Niesytto E. Prognosis in acute lymphoblastic leukemia (ALL) in children preceded by an aplastic phase. Leuk Lymphoma 1994;13(5-6):517-8.

12. Moorman A.V., Ensor H.M., Richards S.M., Chilton L., Schwab C., Kinsey S.E. et al. Prognostic effect of chromosomal abnormalities in childhood B-cell precursor acute lymphoblastic leukaemia: results from the UK Medical Research Council ALL97/99 randomised trial. Lancet Oncol 2010; 11(5):429-38.

13. Lokadasan R., Prem S., Koshy S.M., Jayasudha A.V. Hypercalcaemia with disseminated osteolytic lesions: a rare presentation of childhood acute lymphoblastic leukaemia. Ecancermedicalscience 2015;9:542.

14. Horsley S.W., Colman S., McKinley M., Bateman C.M., Jenney M., Chaplin T. et al. Genetic lesions in a preleukemic aplasia phase in a child with acute lymphoblastic leukemia. Genes Chromosomes Cancer 2008; 47(4):333-40.

15. Urayama K.Y., Buffler P.A., Gallagher E.R., Ayoob J.M., Ma X. A meta-analysis of the association between day-care attendance and childhood acute lymphoblastic leukaemia. Int J Epidemiol 2010; 39(3):718-32.

16. Wegelius R. Bone marrow dysfunctions preceding acute leukemia in children: a clinical study. Leuk Res 1992; 16(1):71-6.

17. Shi J., Shao Z., Liu H., Bai J., Cao Y., He G. et al. Transformation of myelodysplastic syndromes into acute myeloid leukemias. Chin Med J (Engl) 2004; 117(7):963-7.

18. Liang Y., Ding L., Li X., Wang W., Zhang X. Transient pancytopenia preceding adult acute lymphoblastic leukemia with chromosomal abnormalities including the Philadelphia chromosome: A case report and review of the literature. Oncol Lett 2015; 10(6):3789-92.

19. Riccio I., Marcarelli M., Del Regno N., Fusco C., Di Martino M., Savarese R. et al. Musculoskeletal problems in pediatric acute leukemia. J Pediatr Orthop B 2013; 22:264-9.

Conflict of interest: none declared Financial support: none declared 